\title{
MODELO DE PREDICCIÓN DE LA CAPACIDAD AERÓBICA DE FUMADORES Y NO FUMADORES
}

\section{AEROBIC CAPACITY PREDICTION MODEL FOR SMOKERS AND NON-SMOKERS}

\author{
Francisco José Burgos Navarrete ${ }^{1}$
}

\section{RESUMEN}

La Capacidad Aeróbica Relativa (CAR, mlO2/kg-min) es una competencia umbral al considerar personas elegibles para ciertos puestos de trabajo, para medirla indirectamente el investigador de campo tiene limitados equipos portátiles, sencillos y económicos. Este trabajo se justifica por su aporte al rápido y fácil diagnóstico de la CAR, para la correcta ubicación del trabajador sano, en un puesto de trabajo que no supere sus limitaciones, de lo contrario, se dificulta prevenir accidentes y controlar riesgos. El objetivo de esta investigación es modelar funciones multivariadas de regresión, predictoras de la CAR en fumadores y no fumadores, a través de paradigma cuantitativo, diseño bibliográfico, tipo documental, epidemiológico, transversal. El análisis de regresión permitió validar las funciones modeladas, de las cuales se compararon sus resultados versus los obtenidos empleando métodos provenientes de la literatura sobre el tema. Resultados: funciones de regresión, CAR $=\mathrm{A}-0,4806$ (Frecuencia cardíaca reposo) - 0,1978(Edad) + 161,7(Talla) - 1,626(Masa corporal) + 4,29(Índice masa corporal); donde el valor de A corresponde a: Hombres fumadores $=-182,9$; Hombres no fumadores $=-181,6$; mujeres fumadoras $=-182,7 ;$ Mujeres no fumadoras $=-181,4$. Se concluye que con base al coeficiente de determinación R2=86,19\% el modelo propuesto sin ejercicios, es una alternativa aceptable a las mediciones directas.

Palabras claves: Capacidad aeróbica relativa, Frecuencia Cardíaca Reposo, Fumadores y no fumadores, Funciones multivariadas de regresión, Índice masa Corporal, Método sin ejercicios.

\footnotetext{
${ }^{1}$ Profesor Asociado, Universidad Central de Venezuela (UCV), Facultad de Agronomía, Departamento de Química y Tecnología. Maracay, República Bolivariana de Venezuela. orcid.org/0000-0002-2040-549X
} 


\section{ABSTRACT}

Relative Aerobic Capacity (RAC, $\mathrm{mlO} 2 / \mathrm{kg}$-min) is a threshold competence when considering people eligible for certain jobs, to measure it indirectly, the field researcher has limited, simple and inexpensive portable equipment. This work is justified by its contribution to the quick and easy diagnosis of the RAC, for the correct location of the healthy worker, in a job that does not exceed its limitations, otherwise it is difficult to prevent accidents and control risks. Objective: to model multivariate regression functions, predictors of RAC in smokers and non-smokers. Methodology: quantitative paradigm, bibliographic design, documentary, epidemiological, cross-sectional type. The regression analysis allowed validating the modeled functions, from which their results were compared against those obtained using methods from the literature on the subject, Results: regression functions, $R A C=A-0,4806$ (Resting heart rate) - 0,1978(Age) +161,7(Height) - 1,626(Body mass) + 4,29 (Body mass index); where the value of a corresponds to: Male smokers $=-182,9$; Non-smoking men $=-181,6$; female smokers $=-182,7$; Non-smoking women $=-181,4$. Conclusion: a coefficient of determination R2 $=$ $86,19 \%$, indicates that the proposed model without exercises is an acceptable alternative to direct measurement.

Keywords: Relative aerobic capacity, Heart Rate Rest, Smokers and non-smokers; Multivariate regression functions, Body mass index, Exercise-free method.

\section{INTRODUCCIÓN}

La Potencia Aeróbica o Capacidad Aeróbica Relativa (CAR, $\mathrm{mlO}_{2} / \mathrm{kg}-\mathrm{min}$ ), competencia umbral (passing score), capacidad máxima del motor aeróbico, está relacionada con el tamaño corporal y las personas corpulentas presentan valores mayores (Sharkey \& Davis, 2008) y se considera en personas elegibles para ciertos puestos de trabajo.

La CAR se emplea para clasificar el nivel de aptitud de la capacidad cardiorespiratoria (CCR) de un individuo o para comparar los niveles de aptitud de individuos de diferente corpulencia (Heyward, 2010).

Actualmente para medirla indirectamente (método sin ejercicios) el investigador de campo tiene limitados equipos portátiles, sencillos y económicos. En este contexto, Carmona (2003) ha indicado que "mientras no se demuestre lo contrario, la medida directa es la que proporciona los valores más precisos de las variables consideradas...".

Respecto a la actual situación para valorar la CAR, hay laboriosas pruebas empíricas directas de aptitud física (medición de consumo de oxígeno al ejercitarse empleando bicicleta de laboratorio o cintas de correr) Sharkey y Davis (2008) e indirecto como la prueba del escalón de la Forest Service de EE.UU (George, et al. 2007), o en su defecto emplear un método sin ejercicios, para evaluar si los candidatos son idóneos para un puesto específico de trabajo en una determinada organización, y hacer un seguimiento con una evaluación anual del desempeño. Para Heyward (2010), la CAR se emplea para clasificar el nivel de aptitud de la 
capacidad cardiorespiratoria (CCR) de un individuo o para comparar los niveles de aptitud de individuos de diferente corpulencia.

El desconocimiento de la CAR del aspirante a un puesto de trabajo, puede conducir a ubicar al trabajador en puestos que superen sus limitaciones, con una problemática que dificulta el control de riesgos y prevención de accidentes de trabajo y enfermedades ocupacionales, lo cual está tipificado en Ley Orgánica de Prevención, Condiciones y Medio Ambiente de Trabajo (LOPCYMAT, 2005), Artículo 62 numeral 3.

Papathanasiou et al. (2013), concluyen que fumar afecta la Frecuencia Cardíaca (FC) de los jóvenes, aumentando la FC en reposo, desacelerando el aumento de la FC durante el ejercicio y afectando su capacidad para alcanzar la FC máx predicha por edad. Nealen (2016) señala que los sujetos con antecedentes de tabaquismo muestran mayores tasas cardíacas en reposo que aquellos sin historial de tabaquismo, efecto que se debió principalmente a la reducción de horas de ejercicio por los fumadores que al hábito de fumar en sí mismo. De acuerdo con Heyward (2010), una baja Tasa Cardíaca en reposo, ha sido reportada para atletas de alto rendimiento. Con estas investigaciones se evidencia que el tabaquismo se correlaciona con una baja CAR. En este contexto, Whaley et al. (1992), Inbar et al. (1994), Robergs y Landwehr (2002) han reportado modelos para calcular la Frecuencia Cardíaca Máxima. En este contexto, la literatura sobre la valoración de la capacidad aeróbica, pocas veces incluye categorías de Fumadores y No Fumadores. Investigaciones de Strassmann et al. (2013) y Scotland (2012) han reportado influencia en el bajo índice de masa corporal (IMC) de los fumadores respecto a los no fumadores.

La estimación de la CAR $\left(\mathrm{VO}_{2 \text { máx }}\right)$ sin realizar ejercicios, en el presente trabajo se apoya en la bondad demostrada en la Estimación del $\mathrm{VO}_{2 \text { máx }}$ a partir de la relación entre $\mathrm{FC}_{\text {máx }} \mathrm{Y}$ $\mathrm{FC}_{\text {reposo, }}$, fórmula que permite resolver el problema del cálculo de la Potencia Aeróbica o CAR (Uth et al., 2003). Sin embargo, de acuerdo a nuestro conocimiento, para estimar la CAR sin realizar ejercicios en hombres y mujeres fumadores y no fumadores, no se han realizado funciones multivariadas de regresión para predecir la CAR.

El objetivo general de esta investigación, es modelar funciones de regresión predictoras de la CAR, basadas en: la Edad (E), la Frecuencia Cardíaca en Reposo ( $F_{\text {rep }}$ ), la Estatura ( $T$ ), la Masa Corporal (MC), y el Índice de Masa Corporal (IMC), como alternativa a métodos directos o indirectos sin ejercicios.

Los objetivos específicos son: a) construir tablas estratificadas por sexo y condición de fumador ( $F$ ) y no fumador (NF) que categoricen la data de tal modo que permitan obtener las funciones de regresión a ser modeladas y b) para hombres y mujeres, comparar estadísticamente si es igual el IMC de los F y los NF.

\section{METODOLOGÍA Y MATERIALES}

Esta investigación compila data de origen bibliográfico (pooled data), lo que corresponde a un estudio secundario o análisis secundario de data existente según los lineamientos de Follmer y Dowsett (2012). 
- Período de levantamiento del estudio: noviembre 2017 a marzo 2019.

- Usando como referencia, los aportes conceptuales de Palella y Martins (2010), se puede afirmar que el marco metodológico del presente estudio corresponde al: paradigma cuantitativo, diseño bibliográfico, tipo documental, epidemiológico, transversal.

Con fines de estandarización, las funciones de regresión de esta investigación no deben aplicase a lactantes o embarazadas, personas con: calificación y certificación de discapacidad (Ley para las Personas con Discapacidad, 2007), candidatos con un índice de masa corporal (IMC) $\geq 30 \mathrm{~kg} / \mathrm{m}^{2}$; ni aquellas tratadas con anabolizantes y/o beta bloqueadores, tampoco los que consumieren alcohol 24 horas antes de la entrevista/evaluación, o que estén bajo tratamiento o empleen productos, que interfieran en la Frecuencia Cardíaca en Reposo, a excepción del tabaco.

Si el tamaño muestral es pequeño y siendo las muestras independientes provenientes de poblaciones distintas (muestras independientes), las variables de estudio son continuas y en escala ordinal, se puede emplear la prueba no paramétrica de Kruskal-Wallis H (ANOVA One way test), para comparar las medianas de los IMC entre F y NF (Wiedenhöfer,1993).

\section{Definiciones}

- Tabaquismo: enfermedad crónica, que se caracteriza por la adicción a la nicotina. Ésta, al igual que la cocaína, heroína, alcohol, etc., es una droga que crea dependencia e induce aumento de su tolerancia, es decir, se necesitan dosis crecientes para obtener efecto. Su carácter adictivo hace difícil el abandono y mantiene a los fumadores recibiendo grandes cantidades de tóxicos, irritantes, mutágenos y carcinógenos para obtener una pequeña dosis de nicotina (Ministerio de Salud Subsecretaría de Salud Pública de Chile, 2009).

- $\quad$ Fumador: el estudio de 40 hombres, entre 20 y 30 años, no obesos (IMC $<30 \mathrm{Kg} / \mathrm{m}^{2}$ ), sin endocrinopatías ni patologías cardiovasculares y/o renales. Se catalogó como fumador a la persona que para el momento del estudio estaba fumando más de diez cigarrillos al día. En el presente estudio se denomina fumador al usuario de tabaco bajo la modalidad de combustión incompleta en forma de humo y/o de productos sin humo, como el tabaco para mascar, rapé y otros (Villarroel et al., 2004).

- Población objetivo: aquella con características comunes para las cuales serán extensivas las conclusiones de la investigación (Arias, 2012).

- Población disponible o accesible: es la porción finita de la población objetivo a la que se tuvo acceso y de la cual se extrajo la muestra representativa (Arias, 2012). La población accesible que se consideró en los trabajos de investigación consultados, incluye sujetos sanos, mujeres de 16 a 64 años y hombres de 16,2 a 78 años, F y NF.

- Capacidades o competencias umbral o esenciales: son las necesarias para lograr una actuación media o mínimamente adecuada (León Pérez et al., 2007).

- Variables consideradas. Las predictoras o independientes: Edad, Frecuencia Cardíaca en Reposo, Estatura, Masa Corporal e Índice de Masa Corporal; la variable de salida o dependiente es: la Capacidad Aeróbica Relativa. 
- Frecuencia Cardíaca en Reposo $\left(\mathrm{FC}_{\text {reposo }}\right)$ o Resting Heart rate $\left(\mathrm{RH}_{\text {rest }}\right)$ : es la que corresponde a la menor cantidad de sangre que el corazón bombea, es la que necesita una persona al no estar ejercitándose (o no está físicamente activa). Si la persona está sentada o acostada y está tranquila, relajada y no está enferma, la frecuencia cardíaca suele estar entre 60 latidos/minuto y 100 latidos/minuto (American Heart Association, 2019).

Pero una frecuencia cardíaca $<60$ no necesariamente indica un problema médico. Podría ser el resultado de tomar un beta bloqueador. Una frecuencia cardíaca baja también es común para las personas que realizan mucha actividad física o que son muy atléticas. La actividad física moderada generalmente no afecta mucho el pulso en reposo. Si se está en forma, podría cambiar a 40. Una persona menos activa podría tener una frecuencia cardíaca entre 60 y 100. Esto se debe a que el músculo cardíaco tiene que trabajar más para mantener las funciones corporales, haciéndolo más alto (Aladin et al., 2015).

\section{Métodos e instrumentos para medir la Antropometría y Capacidad aeróbica}

- Para medir la estatura, el peso y la masa corporal, se aplicó la ISO 7250:1976 (Mediciones básicas del cuerpo humano para el diseño tecnológico).

- Para medir el Índice de masa corporal se aplicó el estadístico de Quetelet.

- La Frecuencia cardíaca en reposo, se determinó en posición sedente y después de un reposo de diez minutos, empleando un monitor de presión arterial automático (Heyward, 2010).

- La Frecuencia cardiaca máxima y con ella calcular la capacidad aeróbica, se fundamentan en la metodología (descrita por Uth et al., 2003).

- La Frecuencia cardiaca máxima y la Capacidad aeróbica, en este trabajo, se calculan a partir de la edad, explicadas más adelante.

\section{Fórmulas para calcular la Frecuencia Cardíaca Máxima $\left(\mathrm{FC}_{\max }\right)$ o Heart Rate Maxima $\left(\mathrm{HR}_{\max }\right)$}

La Frecuencia Cardíaca Máxima $\left(\mathrm{FC}_{\max }\right)$ en el presente trabajo, se calcula empleando la ecuación univariada de:

a) (Inbar et al., 1994) Donde $\mathrm{n}=1424$; Mean age (range) $=46,7(20-70)$; Regression: se emplea $\mathrm{HR}_{\max }=205,8-(0,685 \mathrm{x}$ age $) ; \mathrm{r}^{2}=0,45 ; \mathrm{S}_{\mathrm{xy}}=6,4$ beats $/$ min y adicionalmente otros autores indican que es la ecuación general más precisa (Robergs \& Landwehr, 2002).

Nota: esta ecuación no discrimina entre hombres y mujeres ni fumadores o no fumadores.

b) Para estimar la Frecuencia Cardíaca Máxima $\left(\mathrm{FC}_{\max }\right)$ o Heart Rate Maxima $\left(\mathrm{HR}_{\max }\right)$, de sujetos fumadores o no fumadores, se emplearon las ecuaciones multivariadas (de Whaley et al., 1992):

$F C_{\text {Max hombres }}=203,9-0,812($ edad $)+0,276\left(F_{\text {Reposo }}\right)-0,084($ Peso corporal $)-4,5 \times$ Código Fumador (1 fumador, 0 no fumador).

$F C_{\text {Max mujeres }}=204,8-0,718($ edad $)+0,162\left(F_{\text {Reposo }}\right)-0,105$ (Peso corporal) $-6,2 \times$ Código Fumador (1 fumador, 0 no fumador). 


\section{Fórmula para calcular la Capacidad Aeróbica Relativa (CAR) o VO ${ }_{2 \max }$}

La Capacidad Aeróbica Relativa (CAR) o $\mathrm{VO}_{2 \max }$ en $\mathrm{mlO}_{2} / \mathrm{kg}$-min, en este trabajo se calcula empleando la ecuación:

$$
\mathrm{VO}_{2 \max }=15,3\left(\mathrm{FC}_{\max } / \mathrm{FC}_{\text {reposo }}\right)
$$

Desarrollada por Uth, et al. (2003).

\section{Predicción de la capacidad aeróbica}

Para generar las funciones de regresión, se usó el software Minitab 17.3, en versión de prueba disponible en la Web.

En este trabajo a la data compilada se le realizó un análisis de Pareto, el cual indicó que el efecto de incluir la variable perímetro abdominal (PA) en la función multivariada de regresión de regresión es muy pequeño, razón por lo que no se tomó en cuenta esta variable independiente para la predicción de la Capacidad Aeróbica Relativa (CAR).

\section{Tratamientos, tamaño muestral, estratificación}

Se condicionó este trabajo a la disponibilidad de tiempo y recursos del investigador acogiéndose al criterio de Ary et al. (1979), la población accesible multiétnica: $n=19196$, fue estratificada en mujeres de 16 a 64 años y hombres de 16,2 a 78 años, en las categorías de F y NF.

\section{Análisis estadístico}

Para constatar si las funciones de regresión modeladas tienen valor práctico, se calcula su coeficiente de determinación $\left(R^{2}\right)$ y se validan sus resultados comparándolos con los generados por otros autores ampliamente aplicadas y aceptadas.

\section{RESULTADOS Y DISCUSIÓN}

En la presente investigación se encontro que hay poca información preliminar documental sobre la CAR y las características antropométricas de la mujer fumadora.

La Tabla 1, resume el modelo de regresión tomando en cuenta todas las observaciones para hombres y mujeres, fumadores y no fumadores.

Para los fines del presente estudio, se considera que los valores de desviación estándar aportan valor agregado, y siendo que los valores promedio son indispensables, es ineludible su presentación y han sido mostrados en las Tablas pertinentes; la data sobre hombres y mujeres, fumadores o no, se presenta por separado.

La data que generó las funciones al emplear el software Minitab 17.3 presentó $\mathbf{R}_{\text {calc }}{ }=86,69 \%=$ 0,8669 ; siendo que $\mathbf{R}_{\text {calc }}=93,10 \%=0,9310>>$ que el valor crítico $R_{\text {tabulado }} \sim 0,46$ indicado en el Anexo 1 (proveniente del texto de Kennedy y Neville (1976) o en la Tabla Valores del Coeficiente 
de Correlación (R) en la Web de: Statistical Manual (Crow et al., 1960), por lo tanto, hay una excelente correlación.

Tabla 1. Resumen del modelo de regresión para los resultados

\begin{tabular}{|c|c|c|c|}
\hline $\mathrm{S}$ & $\mathrm{R}^{2}$ & $\mathrm{R}^{2}$ ajustado & $\mathrm{R}^{2}$ predicho \\
\hline 3,31473 & $86,69 \%$ & $84,38 \%$ & $80,03 \%$ \\
\hline
\end{tabular}

La Tabla 2a, Tabla 3a, Tabla 4a, para hombres y la Tabla 5a, Tabla 6a, Tabla 7a, para mujeres, compilan data primaria: la antropométrica y fisiológica (la capacidad aeróbica).

La Tabla 2b, Tabla 3b, Tabla 4b, para hombres y la Tabla 5b, Tabla 6b, Tabla 7b, para mujeres, compilan resultados que proveen información de valor estadístico sobre: la antropometría y fisiología de los sujetos y las funciones de regresión modeladas.

Las funciones multivariadas de regresión obtenidas en este estudio, están indicadas más adelante discriminadas por medio de una secuencia numérica del (1) al (6) hacia el margen derecho.

Tabla 2a. Data mixta antropométrica y capacidad aeróbica, fumadores y no fumadores

\begin{tabular}{|c|c|c|c|c|c|c|c|c|}
\hline & \multirow{3}{*}{$\begin{array}{c}\mathbf{X 1} \\
\text { Frecuencia } \\
\text { cardíaca } \\
\text { reposo } \\
\text { (latidos/min) } \\
\overline{\boldsymbol{X}}\end{array}$} & \multirow{3}{*}{$\begin{array}{c}\mathbf{X 2} \\
\text { Edad } \\
\text { (años) }\end{array}$} & \multirow{2}{*}{$\frac{\mathbf{X 3}}{\text { Talla }(\mathrm{m})}$} & \multirow{3}{*}{$\begin{array}{c}\mathbf{X 4} \\
\text { Masa } \\
\text { corporal } \\
(\mathrm{kg})\end{array}$} & \multirow{2}{*}{ X5 } & \multirow{3}{*}{$\begin{array}{c}\mathrm{Y} \\
\text { Capacidad } \\
\text { Aeróbica } \\
\text { Relativa } \\
\text { (miO } / \mathrm{mg}_{2} / \mathrm{kg} \text {-min) } \\
\overline{\boldsymbol{X}}\end{array}$} \\
\hline & & & & & & & & \\
\hline HOMBRES & $\mathbf{n}$ & $\begin{array}{c}\text { Frecuencia } \\
\text { cardíaca } \\
\text { máxima } \\
\text { (latidos/min) } \\
\bar{x}\end{array}$ & & & $\bar{x}$ & & $\begin{array}{l}\text { Índice } \\
\text { de masa } \\
\text { corporal } \\
\left(\mathrm{kg} / \mathrm{m}^{2}\right) \\
\overline{\mathcal{X}}\end{array}$ & \\
\hline $\begin{array}{l}\text { Bandyopadhyay } \\
\text { y Bandyopadhyay } \\
(2007)\end{array}$ & 75 & 191,0 * & 69,70 ** & 21,6 & 1,695 & 56,7 & 19,90 & 41,90 \\
\hline $\begin{array}{l}\text { Ekblom-Bak et al. } \\
\text { (2009) }\end{array}$ & 781 & $177,3^{*}$ & 77,00 ** & 41,5 & 1,790 & 80,5 & 25,10 & 35,20 \\
\hline $\begin{array}{l}\text { Secchi y Garcia } \\
\text { (2013) }\end{array}$ & 104 & 192,0 * & 67,00 ** & 20,1 & 1,739 & 71,1 & 23,50 & 43,80 \\
\hline Antolini (2014) & 49 & 178,0 * & 64,50 ** & 40,5 & 1,792 & 89,5 & 27,87 & 42,20 \\
\hline $\begin{array}{l}\text { Pourtaghi et al. } \\
\text { (2014) }\end{array}$ & 12.635 & $189,3^{*}$ & 54,00 ** & 24,0 & 1,741 & 70,0 & 23,30 & 53,60 \\
\hline $\begin{array}{l}\text { Ramírez-Velez et } \\
\text { al. (2014) }\end{array}$ & 21 & 186,6 * & 62,40 ** & 27,9 & 1,656 & 66,3 & 24,10 & 45,90 \\
\hline Rahimi et al. (2017) & 230 & $185,5^{*}$ & 68,30 ** & 29,5 & 1,693 & 73,5 & 25,59 & 41,52 \\
\hline $\begin{array}{l}\text { Fernández et al. } \\
(2018)\end{array}$ & 186 & 191,0 * & 67,98 ** & 21,5 & 1,7143 & 67,6 & 22,99 & 43,00 \\
\hline $\begin{array}{l}\text { Sharma et al. } \\
(2016)\end{array}$ & 30 & 186,0 * & $64,55^{* *}$ & 28,8 & 1,630 & 64,3 & 24,59 & 44,10 \\
\hline \multirow{2}{*}{ Stephens (2004) } & 95 & $190,1^{*}$ & 55,81 ** & 22,9 & 1,804 & 83,1 & 25,00 & 52,11 \\
\hline & 118 & $189,7^{*}$ & $56,35^{* *}$ & 23,5 & 1,808 & 81,2 & 24,80 & 51,50 \\
\hline $\begin{array}{l}\text { Grigaliūnienè et al } \\
(2013)\end{array}$ & $\begin{array}{l}21 \\
24 \\
30 \\
16\end{array}$ & $\begin{array}{l}189,0 \text { * } \\
182,1^{*} \\
175,3^{*} \\
168,4^{*}\end{array}$ & $\begin{array}{l}71,67^{* *} \\
76,04^{* *} \\
81,55^{* *} \\
87,25^{* *}\end{array}$ & $\begin{array}{l}24,5 \\
34,5 \\
44,5 \\
54,5\end{array}$ & $\begin{array}{l}1,820 \\
1,809 \\
1,801 \\
1,741\end{array}$ & $\begin{array}{l}80,9 \\
84,0 \\
85,5 \\
88.6\end{array}$ & $\begin{array}{l}24,44 \\
25,63 \\
26,43 \\
29,21\end{array}$ & $\begin{array}{l}40,35 \\
36,65 \\
32,89 \\
29,54\end{array}$ \\
\hline
\end{tabular}

Estimados empleando: ${ }^{*} \mathrm{FC}_{\max }=205,8-(0,685 \times$ age $) ;{ }^{* *} \mathrm{FC}_{\mathrm{rep}}=15,3\left(\mathrm{HR}_{\max } / \mathrm{CAR}\right)$. 
Tabla 2 b. Resultados de data mixta antropomética y capacidad aeróbica, fumadores y no fumadores

\begin{tabular}{|c|c|c|c|c|c|c|c|c|c|}
\hline $\begin{array}{l}\text { HOMBRES } \\
\text { FUENTE }\end{array}$ & $\mathbf{n}$ & $\begin{array}{c}\text { Frecuencia } \\
\text { cardiaca } \\
\text { máxima } \\
\text { (latidos } / \mathrm{min} \text { ) }\end{array}$ & $\begin{array}{c}\text { Frecuencia } \\
\text { cardiaca } \\
\text { reposo } \\
\text { (latidos/ } \\
\text { min) }\end{array}$ & $\begin{array}{l}\text { Edad } \\
\text { (años) }\end{array}$ & $\begin{array}{l}\text { Talla } \\
(\mathrm{m})\end{array}$ & $\begin{array}{l}\text { Masa } \\
\text { corporal } \\
(\mathrm{kg})\end{array}$ & $\begin{array}{c}\text { Índice } \\
\text { de masa } \\
\text { corporal } \\
\left(\mathrm{kg} / \mathrm{m}^{2}\right)\end{array}$ & Fumador & $\begin{array}{c}\text { Capacidad } \\
\text { Aeróbica } \\
\text { Relativa } \\
\left(\mathrm{mlO}_{2} / \mathrm{kg}-\right. \\
\mathrm{min})\end{array}$ \\
\hline $\begin{array}{l}\text { Bandyopadhyay y } \\
\text { Bandyopadhyay } \\
\text { (2007) }\end{array}$ & 75 & 191 & 69,7 & 21,6 & 1,695 & 56,7 & 19,9 & MIX & 41,9 \\
\hline $\begin{array}{l}\text { Ekblom-Bak et al. } \\
\text { (2009) }\end{array}$ & 781 & 177 & 77,0 & 41,5 & 1,790 & 80,5 & 25,1 & MIX & 35,2 \\
\hline $\begin{array}{l}\text { Secchi y Garcia } \\
(2013)\end{array}$ & 104 & 192 & 67,0 & 20,1 & 1,739 & 71,1 & 23,5 & MIX & 43,8 \\
\hline Antolini (2014) & 49 & 178 & 64,5 & 40,5 & 1,792 & 89,5 & 27,9 & MIX & 42,2 \\
\hline $\begin{array}{l}\text { Pourtaghi et al. } \\
\text { (2014) }\end{array}$ & 12.635 & 189 & 54,0 & 24,0 & 1,741 & 70,0 & 23,3 & MIX & 53,6 \\
\hline $\begin{array}{l}\text { Ramírez-Velez et } \\
\text { al. (2014) }\end{array}$ & 21 & 187 & 62,4 & 27,9 & 1,656 & 66,3 & 24,1 & MIX & 45,9 \\
\hline Rahimi et al. (2017) & 230 & 187 & 68,3 & 29,5 & 1,693 & 73,5 & 25,6 & MIX & 41,5 \\
\hline $\begin{array}{l}\text { Fernández et al. } \\
(2018)\end{array}$ & 186 & 187 & 68,0 & 21,5 & 1,714 & 67,6 & 23,0 & MIX & 43,0 \\
\hline $\begin{array}{l}\text { Shama et al. } \\
(2016)\end{array}$ & 30 & 187 & 64,6 & 28,8 & 1,630 & 64,3 & 24,6 & MIX & 44,1 \\
\hline Stephens (2004) & 95 & 190 & 55,8 & 22,9 & $, 1,804$ & 83,1 & 25,0 & MIX & 52,1 \\
\hline Stepehens (2004) & 118 & 190 & 56,4 & 23,5 & 1,808 & 81,2 & 24,8 & MIX & 51,5 \\
\hline \multirow{4}{*}{$\begin{array}{l}\text { Grigaliüniené et } \\
\text { al. (2013) }\end{array}$} & 21 & 189 & 71,7 & 24,5 & 1,820 & 80,9 & 24,4 & MIX & 40,4 \\
\hline & 24 & 182 & 76,0 & 34,5 & 1,809 & 84,0 & 25,6 & MIX & 36,7 \\
\hline & 30 & 175 & 81,6 & 44,5 & 1,801 & 85,5 & 26,4 & MIX & 32,9 \\
\hline & 16 & 168 & 87,3 & 54,5 & 1,741 & 88,6 & 29,2 & MIX & 29,5 \\
\hline \multirow{5}{*}{\multicolumn{2}{|c|}{$\sum n=14.415$}} & MEDIA & 68,3 & 30,7 & 1,7 & 76,2 & 24,8 & & 42,3 \\
\hline & & MEDIANA & 68,0 & 27,9 & 1,7 & 80,5 & 24,8 & & 42,2 \\
\hline & & MÁXIMO & 87,3 & 54,5 & 1,8 & 89,5 & 29,2 & & 53,6 \\
\hline & & MÍNIMO & 54,0 & 20,1 & 1,6 & 56,7 & 19,9 & & 29,5 \\
\hline & & $\begin{array}{l}\text { DESV. } \\
\text { ESTANDAR }\end{array}$ & 9,5 & 10,3 & 0,1 & 9,8 & 2,2 & & 6,9 \\
\hline
\end{tabular}

\section{Función de regresión Tabla $2 \mathrm{~b}$ - Hombres MIX}

Capacidad Aeróbica Relativa $\left(\mathrm{mlO}_{2} / \mathrm{kg}-\mathrm{min}\right)=$

-183,4 - 0,4806 (Frecuencia cardíaca reposo, lat/min) - 0,1978 (Edad, años) + 161,7 (Talla, m)

- 1,626 (Masa corporal, kg) + 4,29 (Índice de masa corporal, kg/m²). (1) 
Tabla 3a. Data antropométrica y capacidad aeróbica, fumadores

\begin{tabular}{|c|c|c|c|c|c|c|c|c|}
\hline & & & $\mathrm{X} 1$ & $\mathrm{X} 2$ & $\mathrm{X} 3$ & $\mathrm{X} 4$ & $\times 5$ & $\mathbf{Y}$ \\
\hline HOMBRES & $n$ & $\begin{array}{c}\text { Frecuencia } \\
\text { cardíaca } \\
\text { máxima (latidos/ } \\
\text { min) } \\
\bar{x}\end{array}$ & $\begin{array}{l}\text { Frecuencia } \\
\text { cardíaca } \\
\text { reposo (latidos/ } \\
\text { min) } \\
\overline{\mathcal{X}}\end{array}$ & $\begin{array}{l}\text { Edad } \\
\text { (años) } \\
\bar{x}\end{array}$ & $\begin{array}{l}\text { Talla (m) } \\
\qquad \bar{x}\end{array}$ & $\begin{array}{c}\text { Masa } \\
\text { corporal } \\
(\mathrm{kg}) \\
\overline{\boldsymbol{x}}\end{array}$ & $\begin{array}{c}\text { Índice } \\
\text { de masa } \\
\text { corporal }(\mathrm{kg} / \\
\frac{\left.\mathrm{m}^{2}\right)}{\bar{x}}\end{array}$ & $\begin{array}{c}\text { Capacidad } \\
\text { Aeróbica } \\
\text { Relativa } \\
\text { (mlO } / \\
\text { kg-min) } \\
\bar{x}\end{array}$ \\
\hline $\begin{array}{l}\text { Miyatake et. } \\
\text { al.(2012) }\end{array}$ & 149 & $177,6^{*}$ & 71,00 & 44,1 & 1,702 & 66,40 & 22,90 & 37,20 \\
\hline $\begin{array}{l}\text { Paracha et } \\
\text { al. (2015) }\end{array}$ & 100 & $175,1^{*}$ & 69,00 & 45,8 & 1,683 & 73,12 & 25,77 & 38,82 ** \\
\hline \multirow{4}{*}{$\begin{array}{l}\text { Eroğlu y } \\
\text { Yüksek } \\
\text { (2018) }\end{array}$} & 69 & 163,9 * & 75,80 & 61,6 & 1,702 & 75,30 & 26,00 & 33,09 ** \\
\hline & 87 & 159,9 * & 75,70 & 66,5 & 1,707 & 75,80 & 26,00 & 32,32 ** \\
\hline & 45 & $156,1^{*}$ & 75,70 & 71,6 & 1,685 & 72,00 & 25,30 & $31,55^{* *}$ \\
\hline & 38 & 151,2 * & 74,30 & 77,2 & 1,691 & 71,40 & 25,10 & 31,14 ** \\
\hline $\begin{array}{l}\text { Shephard y } \\
\text { Pimm (1975) }\end{array}$ & 10 & $190,7^{*}$ & 60,72 & 22,9 & 1,775 & 81,20 & 25,77 & $48,06^{* *}$ \\
\hline
\end{tabular}

\section{Estimados empleando:}

* $\quad$ FC $_{\text {Max hombres }}=203,9-0,812\left(\right.$ edad) $+0,276\left(F_{\text {Reposo }}\right)-0,084$ (Peso corporal) $-4,5 \times$ Código

Fumador (1 fumador, 0 no fumador); ${ }^{*}$ CAR $=15,3\left(\mathbf{H R}_{\max } / \mathrm{FC}_{\text {rep }}\right)$.

Tabla 3b. Resultados de data mixta antropomética y capacidad aeróbica, fumadores

\begin{tabular}{|c|c|c|c|c|c|c|c|c|c|}
\hline $\begin{array}{l}\text { HOMBRES } \\
\text { FUENTE }\end{array}$ & $\mathbf{n}$ & $\begin{array}{c}\text { Frecuencia } \\
\text { cardiaca } \\
\text { máxima } \\
\text { (latidos/ } \\
\text { min) }\end{array}$ & $\begin{array}{c}\text { Frecuencia } \\
\text { cardiaca } \\
\text { reposo } \\
\text { (latidos/ } \\
\text { min) }\end{array}$ & $\begin{array}{l}\text { Edad } \\
\text { (años) }\end{array}$ & $\begin{array}{l}\text { Talla } \\
(\mathrm{m})\end{array}$ & $\begin{array}{l}\text { Masa } \\
\text { corporal } \\
(\mathrm{kg})\end{array}$ & $\begin{array}{c}\text { Índice } \\
\text { de masa } \\
\text { corporal } \\
\left(\mathrm{kg} / \mathrm{m}^{2}\right)\end{array}$ & Fumador & $\begin{array}{c}\text { Capacidad } \\
\text { Aeróbica } \\
\text { Relativa } \\
\left(\mathrm{mlO}_{2} / \mathrm{kg}-\right. \\
\mathrm{min})\end{array}$ \\
\hline Miyatake et al. (2012) & 149 & 178 & 71,0 & 44,1 & 1,702 & 66,4 & 22,9 & $\mathrm{~F}$ & 37,2 \\
\hline Paracha et.al. (2015) & 100 & 175 & 69,0 & 45,8 & 1,683 & 73,1 & 25,8 & $\mathrm{~F}$ & 38,8 \\
\hline \multirow[t]{4}{*}{ Eroğlu y Yüksek (2018) } & 69 & 164 & 75,8 & 61,6 & 1,702 & 75,3 & 26,0 & $\mathrm{~F}$ & 33,1 \\
\hline & 87 & 160 & 75,7 & 66,5 & 1,707 & 75,8 & 26,0 & $\mathrm{~F}$ & 32,3 \\
\hline & 45 & 156 & 75,7 & 71,6 & 1,685 & 72,0 & 25,3 & $\mathrm{~F}$ & 31,6 \\
\hline & 38 & 151 & 74,3 & 77,2 & 1,691 & 71,4 & 25,1 & $\mathrm{~F}$ & 31,1 \\
\hline Shephard y Pimm (1975) & 10 & 191 & 60,7 & 22,9 & 1,775 & 81,2 & 25,8 & $\mathrm{~F}$ & 48,1 \\
\hline \multirow{5}{*}{\multicolumn{2}{|c|}{$\sum n=498$}} & MEDIA & 71,7 & 55,7 & 1,7 & 73,6 & 25,3 & & 36,0 \\
\hline & & MEDIANA & 74,3 & 61,6 & 1,7 & 73,1 & 25,8 & & 33,1 \\
\hline & & MÁXIMO & 75,8 & 77,2 & 1,8 & 81,2 & 26,0 & & 48,1 \\
\hline & & MÍNIMO & 60,7 & 22,9 & 1,7 & 66,4 & 22,9 & & 31,1 \\
\hline & & $\begin{array}{l}\text { DESV. } \\
\text { ESTANDAR }\end{array}$ & 5,5 & 19,0 & 0,0 & 4,6 & 1,1 & & 6,1 \\
\hline
\end{tabular}

\section{Función de regresión Tabla 3b - HOMBRES FUMADORES}

Capacidad aeróbica relativa $\left(\mathrm{mlO}_{2} / \mathrm{kg}-\mathrm{min}\right)=$

-182,9 - 0,4806 (Frecuencia cardíaca reposo, lat/min) - 0,1978 (Edad, años) + 161,7 (Talla, m)

- 1,626 (Masa corporal, $\mathrm{kg}$ ) + 4,29 (Índice de masa corporal, $\mathrm{kg} / \mathrm{m}^{2}$ ). 
Tabla 4a. Data antropométrica y capacidad aeróbica, no fumadores

\begin{tabular}{|c|c|c|c|c|c|c|c|c|}
\hline & & & $\mathrm{X1}$ & $\mathrm{X} 2$ & $\mathrm{X3}$ & $\mathrm{X} 4$ & $\times 5$ & $\bar{Y}$ \\
\hline HOMBRES & $n$ & $\begin{array}{l}\text { Frecuencia } \\
\text { cardíaca } \\
\text { máxima } \\
\text { (latidos/min) } \\
\quad \bar{x}\end{array}$ & $\begin{array}{l}\text { Frecuencia } \\
\text { cardíaca } \\
\text { reposo } \\
\text { (latidos/min) } \\
\quad \bar{x}\end{array}$ & $\begin{array}{c}\text { Edad } \\
\text { (años) } \\
\bar{x}\end{array}$ & $\begin{array}{l}\text { Talla } \\
(\mathrm{m}) \\
\bar{x}\end{array}$ & $\begin{array}{c}\text { Masa } \\
\text { corporal } \\
(\mathrm{kg}) \\
\overline{\boldsymbol{x}}\end{array}$ & $\begin{array}{c}\text { Índice } \\
\text { de masa } \\
\text { corporal } \\
\left(\mathrm{kg} / \mathrm{m}^{2}\right) \\
\bar{x}\end{array}$ & $\begin{array}{c}\text { Capacidad } \\
\text { aeróbica relativa } \\
\left(\mathrm{mlO}_{2} / \mathrm{kg}-\mathrm{min}\right) \\
\overline{\mathcal{X}}\end{array}$ \\
\hline Paracha et al. (2015) & 99 & $177,8^{*}$ & 67,00 & 46,93 & 1,688 & 76,17 & 26,68 & 40,62 ** \\
\hline \multirow{4}{*}{$\begin{array}{l}\text { Eroğlu y Yüksek, } \\
\text { (2018) }\end{array}$} & 74 & 168,0 * & 75,30 & 61,50 & 1,703 & 79,70 & 27,30 & $34,14^{* *}$ \\
\hline & 93 & $164,3^{*}$ & 75,30 & 66,40 & 1,687 & 76,30 & 26,80 & 33,39 ** \\
\hline & 90 & 159,9 * & 73,90 & 71,40 & 1,681 & 76,20 & 27,00 & 33,10 ** \\
\hline & 68 & 153,4 * & 72,60 & 78,80 & 1,673 & 76,90 & 27,50 & 32,34 ** \\
\hline Choi et al. (2016) & 342 & $178,1^{*}$ & 58,8 & 42,40 & 1,796 & 89,5 & 27,7 & $46,20^{\star *}$ \\
\hline $\begin{array}{l}\text { Shephard y Pimm } \\
\text { (1975) }\end{array}$ & 68 & $194,3^{*}$ & 56,00 & 22,90 & 1,773 & 76,50 & 24,33 & $53,09^{* *}$ \\
\hline $\begin{array}{l}\text { Santos Silva et al. } \\
\text { (2018) }\end{array}$ & 695 & $194,7^{*}$ & 76,10 & 16,20 & 1,727 & 65,70 & 22,00 & 42,70 \\
\hline Ruiz et al. (2017) & 8 & $189,2^{*}$ & 58,70 & 28,70 & 1,790 & 89,30 & 27,60 & 49,80 \\
\hline \multirow{4}{*}{ Ogawa et al. (1992) } & 14 & 196,0 * & 72,00 & 27,00 & 1,768 & 75,30 & 24,00 & 45,90 \\
\hline & 13 & 166,0 * & 75,00 & 63,50 & 1,765 & 83,40 & 26,70 & 27,20 \\
\hline & 15 & 192,0 * & 61,00 & 28,00 & 1,796 & 68,40 & 21,20 & 63,50 \\
\hline & 14 & 164,0 * & 60,00 & 63,00 & 1,734 & 65,90 & 21,90 & 47,60 \\
\hline
\end{tabular}

\section{Estimados empleando:}

* $\quad$ FCMax hombres $=203,9-0,812$ (edad) + 0,276(FCReposo) - 0,084(Peso corporal) $-4,5 x$ Código Fumador (1 fumador, 0 no fumador); ${ }^{*}$ CAR = 15,3(HRmax/ FCrep). 
Tabla 4b. Resultados de data mixta antropomética y capacidad aeróbica, fumadores

\begin{tabular}{|c|c|c|c|c|c|c|c|c|c|}
\hline $\begin{array}{l}\text { HOMBRES } \\
\text { FUENTE }\end{array}$ & $\mathbf{n}$ & $\begin{array}{c}\text { Frecuencia } \\
\text { cardiaca } \\
\text { máxima } \\
\text { (latidos } / \mathrm{min} \text { ) }\end{array}$ & $\begin{array}{c}\text { Frecuencia } \\
\text { cardiaca } \\
\text { reposo } \\
\text { (latidos/ } \\
\text { min) }\end{array}$ & $\begin{array}{l}\text { Edad } \\
\text { (años) }\end{array}$ & $\begin{array}{l}\text { Talla } \\
(\mathrm{m})\end{array}$ & $\begin{array}{c}\text { Masa } \\
\text { corporal } \\
(\mathrm{kg})\end{array}$ & $\begin{array}{c}\text { Índice } \\
\text { de masa } \\
\text { corporal } \\
\left(\mathrm{kg} / \mathrm{m}^{2}\right)\end{array}$ & Fumador & $\begin{array}{c}\text { Capacidad } \\
\text { Aeróbica } \\
\text { Relativa } \\
\left(\mathrm{mlO}_{2} / \mathrm{kg}-\right. \\
\mathrm{min})\end{array}$ \\
\hline $\begin{array}{l}\text { Paracha et.al. } \\
(2015)\end{array}$ & 99 & 178 & 67,0 & 46,9 & 1,688 & 76,2 & 26,7 & NF & 40,6 \\
\hline \multirow{4}{*}{$\begin{array}{l}\text { Eroğlu y Yüksek } \\
\text { (2018) }\end{array}$} & 74 & 168 & 75,3 & 61,5 & 1,703 & 79,7 & 27,3 & $\mathrm{NF}$ & 34,1 \\
\hline & 93 & 164 & 75,3 & 66,4 & 1,687 & 76,3 & 26,8 & $\mathrm{NF}$ & 33,4 \\
\hline & 90 & 160 & 73,9 & 71,4 & , 1,681 & 76,2 & 27,0 & NF & 33,1 \\
\hline & 68 & 153 & 72,6 & 78,8 & 1,673 & 76,9 & 27,5 & NF & 32,3 \\
\hline Choi et al. (2016) & 342 & 178 & 58,8 & 42,4 & 1,796 & 89,5 & 27,7 & $\mathrm{NF}$ & 46,2 \\
\hline $\begin{array}{l}\text { Shephard y Pimm } \\
\text { (1975) }\end{array}$ & 68 & 194 & 56,0 & 22,9 & 1,773 & 76,5 & 24,3 & NF & 53,1 \\
\hline $\begin{array}{l}\text { Santos Silva et al. } \\
(2018)\end{array}$ & 695 & 195 & 76,1 & 16,2 & 1,727 & 65,7 & 22,0 & NF & 42,7 \\
\hline Ruiz et al. (2019) & 8 & 189 & 58,7 & 28,7 & 1,790 & 89,3 & 27,6 & $\mathrm{NF}$ & 49,8 \\
\hline \multirow{4}{*}{$\begin{array}{l}\text { Ogawa et al. } \\
\text { (1992) }\end{array}$} & 14 & 196 & 72,0 & 27,0 & 1,768 & 75,3 & 24,0 & $\mathrm{NF}$ & 45,9 \\
\hline & 13 & 166 & 75,0 & 63,5 & 1,765 & 83,4 & 26,7 & $\mathrm{NF}$ & 27,2 \\
\hline & 15 & 192 & 61,0 & 28,0 & 1,796 & 68,4 & 21,2 & $\mathrm{NF}$ & 63,5 \\
\hline & 14 & 164 & 60,0 & 63,0 & 1,734 & 65,9 & 21,9 & $\mathrm{NF}$ & 47,6 \\
\hline \multirow{5}{*}{\multicolumn{2}{|c|}{$\sum n=1.593$}} & MEDIA & 67,8 & 47,4 & 1,7 & 76,9 & 25,4 & & 42,3 \\
\hline & & MEDIANA & 72,0 & 46,9 & 1,7 & 76,3 & 26,7 & & 42,7 \\
\hline & & MÁXIMO & 76,1 & 78,8 & 1,8 & 89,5 & 27,7 & & 63,5 \\
\hline & & MÍNIMO & 56,0 & 16,2 & 1,7 & 65,7 & 21,2 & & 27,2 \\
\hline & & $\begin{array}{l}\text { DESV. } \\
\text { ESTANDAR }\end{array}$ & 7,8 & 21,1 & 0,0 & 7,6 & 2,4 & & 10,2 \\
\hline
\end{tabular}

\section{Función de regresión Tabla 4b - HOMBRES NO FUMADORES}

Capacidad aeróbica relativa $\left(\mathrm{mlO}_{2} / \mathrm{kg}-\mathrm{min}\right)=$

-181,6 - 0,4806 (Frecuencia cardíaca reposo, lat/min) - 0,1978 (Edad, años) + 161,7 (Talla, m) - 1,626 (Masa corporal, kg) + 4,29 (Índice de masa corporal, kg/m²) (3) 
Tabla 5a. Data mixta antropométrica y capacidad aeróbica, fumadoras y no fumadoras

\begin{tabular}{|c|c|c|c|c|c|c|c|c|}
\hline & & & & & & \\
\hline & & & $\mathrm{X} 1$ & $\mathrm{X} 2$ & $\mathrm{X} 3$ & $\mathrm{X} 4$ & $\mathrm{X} 5$ & $\mathbf{Y}$ \\
\hline MUJERES & $n$ & $\begin{array}{c}\text { Frecuencia } \\
\text { cardíaca } \\
\text { máxima (latidos/ } \\
\frac{\min }{\bar{x}}\end{array}$ & $\begin{array}{c}\text { Frecuencia } \\
\text { cardíaca reposo } \\
\text { (latidos/min) } \\
\bar{x}\end{array}$ & $\begin{array}{l}\text { Edad } \\
\text { (años) } \\
\bar{x}\end{array}$ & $\begin{array}{l}\text { Talla (m) } \\
\qquad \bar{x}\end{array}$ & $\begin{array}{l}\text { Masa } \\
\text { corporal (kg) } \\
\qquad \bar{x}\end{array}$ & $\begin{array}{c}\text { Índice } \\
\text { de masa } \\
\text { corporal (kg/ } \\
\left.\mathrm{m}^{2}\right) \\
\overline{\boldsymbol{x}}\end{array}$ & $\begin{array}{l}\text { Capacidad } \\
\text { aeróbica } \\
\text { relativa } \\
\left(\mathrm{mlO}_{2} / \mathrm{kg} \text {-min) }\right. \\
\bar{x}\end{array}$ \\
\hline $\begin{array}{l}\text { Ekblom-Bak et } \\
\text { al. (2009) }\end{array}$ & 890 & 177,0 * & $79.69^{* *}$ & 41,9 & 1,657 & 66,1 & 24,10 & 34,00 \\
\hline $\begin{array}{l}\text { Secchi y Garcia } \\
\text { (2013) }\end{array}$ & 136 & 192,3 * & 80,60 ** & 19,6 & 1,631 & 60,7 & 22,80 & 36,50 \\
\hline $\begin{array}{l}\text { González } \\
\text { y Achiardi } \\
(2016)\end{array}$ & 31 & 191,8 * & 86,50 ** & 20,3 & 1,600 & 59,2 & 22,95 & 33,93 \\
\hline \multirow{2}{*}{$\begin{array}{l}\text { Barrios et al. } \\
(2018)\end{array}$} & 118 & 189,0 * & 74,90 ** & 24,3 & 1,620 & 73,0 & 27,83 & 38,62 \\
\hline & 135 & 191,2 * & 64,30 ** & 21,2 & 1.595 & 66,6 & 26,21 & 45,50 \\
\hline $\begin{array}{l}\text { Grigaliūnienè } \\
\text { et al. (2013) }\end{array}$ & $\begin{array}{l}21 \\
18 \\
20 \\
18\end{array}$ & $\begin{array}{l}189,0 \\
182,1 \\
175.3 \\
168,4\end{array}$ & $\begin{array}{c}83,39^{* *} \\
101,82^{* *} \\
105,85^{* *} \\
103,18^{* *}\end{array}$ & $\begin{array}{l}24,5 \\
34,5 \\
44,5 \\
54,5\end{array}$ & $\begin{array}{l}1,690 \\
1,672 \\
1,656 \\
1,644\end{array}$ & $\begin{array}{l}62,14 \\
64,61 \\
65,95 \\
71,44\end{array}$ & $\begin{array}{l}21,78 \\
23,13 \\
24,06 \\
26,91\end{array}$ & $\begin{array}{l}34,68 \\
27,37 \\
25,34 \\
24,98\end{array}$ \\
\hline $\begin{array}{l}\text { Dehneh et al. } \\
\text { (2016) }\end{array}$ & 206 & 190,8 & 79,56 ** & 21,89 & 1,626 & 60,47 & 23,04 & 36,70 \\
\hline \multirow{2}{*}{$\begin{array}{l}\text { Sengupta y } \\
\text { Krajewska- } \\
\text { Kulak (2014) }\end{array}$} & 50 & 191,2 * & 78,60 & 21,3 & 1,564 & 54,3 & 22,2 & 31,00 \\
\hline & 50 & $189,5^{*}$ & 84,10 & 23,7 & 1,578 & 48,8 & 19,60 & 35,00 \\
\hline $\begin{array}{l}\text { Fernández et } \\
\text { al. (2018) }\end{array}$ & 204 & 190,9 * & 86,00 ** & 21,7 & 1,595 & 58,0 & 22,80 & 34,00 \\
\hline
\end{tabular}

Estimados empleando: * HRmax $=205,8-(0,685 x$ age $) ;{ }^{* *}$ FCrep $=15,3($ HRmax/CAR). 
Tabla 5b. Resultados de data mixta antropomética y capacidad aeróbica, fumadoras y no fumadoras

\begin{tabular}{|c|c|c|c|c|c|c|c|c|c|}
\hline $\begin{array}{l}\text { MUJERES } \\
\text { FUENTE }\end{array}$ & $\mathrm{n}$ & $\begin{array}{l}\text { Frecuencia } \\
\text { cardiaca } \\
\text { máxima } \\
\text { (latidos } / \mathrm{min} \text { ) }\end{array}$ & $\begin{array}{l}\text { Frecuencia } \\
\text { cardiaca } \\
\text { reposo } \\
\text { (latidos/ } \\
\text { min) }\end{array}$ & $\begin{array}{l}\text { Edad } \\
\text { (años) }\end{array}$ & $\begin{array}{l}\text { Talla } \\
(\mathrm{m})\end{array}$ & $\begin{array}{c}\text { Masa } \\
\text { corporal } \\
(\mathrm{kg})\end{array}$ & $\begin{array}{l}\text { Índice } \\
\text { de masa } \\
\text { corporal } \\
\left(\mathrm{kg} / \mathrm{m}^{2}\right)\end{array}$ & Fumador & $\begin{array}{l}\text { Capacidad } \\
\text { Aeróbica } \\
\text { Relativa } \\
\left(\mathrm{mlO}_{2} / \mathrm{kg}-\right. \\
\mathrm{min})\end{array}$ \\
\hline $\begin{array}{l}\text { Ekblom-Bak } \\
\text { et al. (2009) }\end{array}$ & 890 & 177 & 79,7 & 41,9 & 1,657 & 66,1 & 24,1 & MIX & 34,0 \\
\hline $\begin{array}{l}\text { Secchi y } \\
\text { Garcia (2013) }\end{array}$ & 136 & 192 & 80,6 & 19,6 & 1,631 & 60,7 & 22,8 & MIX & 36,5 \\
\hline $\begin{array}{l}\text { Gonzàlez y } \\
\text { Achiardi (2016) }\end{array}$ & 31 & 192 & 86,5 & 20,3 & 1,600 & 59,2 & 23,0 & MIX & 33,9 \\
\hline \multirow{2}{*}{$\begin{array}{l}\text { Barrios } \\
\text { Vergara et al. } \\
(2018)\end{array}$} & 118 & 189 & 74,9 & 24,3 & 1,620 & 73,0 & 27,8 & MIX & 38,6 \\
\hline & 135 & 191 & 64,3 & 21,2 & 1,595 & 66,6 & 26,2 & MIX & 45,5 \\
\hline \multirow{4}{*}{$\begin{array}{l}\text { Grigaliüniené } \\
\text { et al. (2013) }\end{array}$} & 21 & 189 & 83,4 & 24,5 & 1,690 & 62,1 & 21,8 & MIX & 34,7 \\
\hline & 18 & 182 & 101,8 & 34,5 & 1,672 & 64,6 & 23,1 & MIX & 27,4 \\
\hline & 20 & 175 & 105,9 & 44,5 & 1,656 & 66,0 & 24,1 & MIX & 25,3 \\
\hline & 18 & 168 & 103,2 & 54,5 & 1,644 & 71,4 & 26,9 & MIX & 25,0 \\
\hline $\begin{array}{l}\text { Dehneh et al. } \\
\text { (2016) }\end{array}$ & 206 & 191 & 79,6 & 21,9 & 1,626 & 60,5 & 23,0 & MIX & 36,7 \\
\hline \multirow{2}{*}{$\begin{array}{l}\text { Segupta y } \\
\text { Krajewska- } \\
\text { Kulak (2014) }\end{array}$} & 50 & 191 & 78,6 & 21,3 & 1,564 & 54,3 & 22,2 & MIX & 31,0 \\
\hline & 50 & 190 & 84,1 & 23,7 & 1,578 & 48,8 & 19,6 & MIX & 35,0 \\
\hline $\begin{array}{l}\text { Fernández et } \\
\text { al. (2018) }\end{array}$ & 204 & 191 & 86,0 & 21,7 & 1,595 & 58,0 & 22,8 & MIX & 34,0 \\
\hline \multirow{5}{*}{\multicolumn{2}{|c|}{$\sum n=1897$}} & MEDIA & 85,3 & 28,8 & 1,6 & 62,4 & 23,6 & & 33,7 \\
\hline & & MEDIANA & 83,4 & 23,7 & 1,6 & 62,1 & 23,0 & & 34,0 \\
\hline & & MÁXIMO & 105,9 & 54,5 & 1,7 & 73,0 & 27,8 & & 45,5 \\
\hline & & MÍNIMO & 64,3 & 19,6 & 1,6 & 48,8 & 19,6 & & 25,0 \\
\hline & & $\begin{array}{l}\text { DESV. } \\
\text { ESTANDAR }\end{array}$ & 11,9 & 11,3 & 0,0 & 6,6 & 2,2 & & 5,6 \\
\hline
\end{tabular}

\section{Función de regresión Tabla $5 b$ - MUJERES MIX}

Capacidad aeróbica relativa $\left(\mathrm{mlO}_{2} / \mathrm{kg}-\mathrm{min}\right)=$

-183,2 - 0,4806 (Frecuencia cardíaca reposo, lat/min) - 0,1978 (Edad, años) + 161,7 (Talla, m)

- 1,626 (Masa corporal, kg) + 4,29 (Índice de masa corporal, kg/m²) (4) 
Tabla 6a. Data antropométrica y capacidad aeróbica, fumadoras

\begin{tabular}{|c|c|c|c|c|c|c|c|c|}
\hline & & & X1 & $\mathrm{X} 2$ & $\mathrm{X} 3$ & $\mathrm{X} 4$ & $X 5$ & $Y$ \\
\hline MUJERES & $n$ & $\begin{array}{l}\text { Frecuencia } \\
\text { cardíaca } \\
\text { máxima } \\
\text { (latidos/min) } \\
\qquad \overline{\mathcal{X}}\end{array}$ & $\begin{array}{l}\text { Frecuencia } \\
\text { cardíaca } \\
\text { reposo } \\
\text { (latidos/min) } \\
\overline{\bar{X}}\end{array}$ & $\begin{array}{l}\text { Edad } \\
\text { (años) } \\
\bar{x}\end{array}$ & $\begin{array}{l}\text { Talla } \\
(\mathrm{m})\end{array}$ & $\begin{array}{c}\text { Masa } \\
\text { corporal } \\
(\mathrm{kg}) \\
\overline{\boldsymbol{x}}\end{array}$ & $\begin{array}{c}\text { Índice } \\
\text { de masa } \\
\text { corporal }(\mathrm{kg} / \\
\frac{\left.\mathrm{m}^{2}\right)}{\bar{X}}\end{array}$ & $\begin{array}{c}\text { Capacidad } \\
\text { aeróbica relativa } \\
\left(\mathrm{mlO}_{2} / \mathrm{kg}-\mathrm{min}\right) \\
\bar{x}\end{array}$ \\
\hline $\begin{array}{l}\text { Shephard y } \\
\text { Pimm (1975) }\end{array}$ & 7 & 188,48 * & 72,89 & 21,6 & 1,683 & 61,10 & 22,09 & 46,03 \\
\hline
\end{tabular}

Estimados empleando: * FC ${ }_{\text {Max mujeres }}=204,8-0,718$ (edad) + 0,162( FC $\left._{\text {Reposo }}\right)-$ 0,105(Peso corporal) - 6,2 x Código Fumador (1 fumador, 0 no fumador).

Tabla 6b. Resultados de data mixta antropomética y capacidad aeróbica, fumadoras y no fumadoras

\begin{tabular}{|c|c|c|c|c|c|c|c|c|c|}
\hline $\begin{array}{l}\text { MUJERES } \\
\text { FUENTE }\end{array}$ & $\mathbf{n}$ & $\begin{array}{c}\text { Frecuencia } \\
\text { cardiaca } \\
\text { máxima } \\
\text { (latidos } / \text { min) }\end{array}$ & $\begin{array}{c}\text { Frecuencia } \\
\text { cardiaca } \\
\text { reposo } \\
\text { (latidos } / \text { min) }\end{array}$ & $\begin{array}{l}\text { Edad } \\
\text { (años) }\end{array}$ & $\begin{array}{l}\text { Talla } \\
(\mathrm{m})\end{array}$ & $\begin{array}{l}\text { Masa } \\
\text { corporal } \\
(\mathrm{kg})\end{array}$ & $\begin{array}{c}\text { Índice } \\
\text { de masa } \\
\text { corporal } \\
\left(\mathrm{kg} / \mathrm{m}^{2}\right)\end{array}$ & Fumador & $\begin{array}{c}\text { Capacidad } \\
\text { Aeróbica } \\
\text { Relativa } \\
\left(\mathrm{mlO}_{2} / \mathrm{kg}-\right. \\
\mathrm{min})\end{array}$ \\
\hline $\begin{array}{l}\text { Shephard y } \\
\text { Primm (1975) }\end{array}$ & 7 & 188 & 72,9 & 21,6 & 1,683 & 61,1 & 22,1 & $\mathrm{~F}$ & 46,0 \\
\hline
\end{tabular}

$\sum n=7$

\section{Función de regresión Tabla 6b - MUJERES FUMADORAS}

Capacidad aeróbica relativa $\left(\mathrm{mlO}_{2} / \mathrm{kg}-\mathrm{min}\right)=$

-182,7 - 0,4806 (Frecuencia cardíaca reposo, lat/min) - 0,1978 (Edad, años) + 161,7 (Talla, m) - 1,626 (Masa corporal, kg) + 4,29 (Índice de masa corporal, kg/m²) (5)

Tabla 7a. Data antropométrica y capacidad aeróbica, no fumadoras

\begin{tabular}{|c|c|c|c|c|c|c|c|c|}
\hline & & & & & & \\
\hline & & & $\mathrm{X} 1$ & $\mathrm{X} 2$ & $\mathrm{X} 3$ & $\mathrm{X} 4$ & $\mathrm{X5}$ & $\mathbf{Y}$ \\
\hline MUJERES & $\mathrm{n}$ & $\begin{array}{l}\text { Frecuencia car- } \\
\text { díaca máxima } \\
\text { (latidos/min) } \\
\bar{x}\end{array}$ & $\begin{array}{l}\text { Frecuencia } \\
\text { cardíaca } \\
\text { reposo (latidos/ } \\
\text { min) } \\
\overline{\boldsymbol{X}}\end{array}$ & $\begin{array}{l}\text { Edad } \\
\text { (años) } \\
\overline{\boldsymbol{x}}\end{array}$ & $\begin{array}{l}\text { Talla } \\
(\mathrm{m}) \\
\bar{x}\end{array}$ & $\begin{array}{c}\text { Masa cor- } \\
\text { poral }(\mathrm{kg}) \\
\overline{\boldsymbol{X}}\end{array}$ & $\begin{array}{c}\text { Índice } \\
\text { de masa } \\
\text { corporal }(\mathrm{kg} / \\
\frac{\left.\mathrm{m}^{2}\right)}{\bar{x}}\end{array}$ & $\begin{array}{c}\text { Capacidad } \\
\text { aeróbica relativa } \\
\left(\mathrm{mlO}_{2} / \mathrm{kg} \text {-min }\right) \\
\overline{\mathcal{X}}\end{array}$ \\
\hline $\begin{array}{l}\text { Santos Silva et } \\
\text { al (2018) }\end{array}$ & 695 & 194,8 & 81,10 & 16,0 & 1,613 & 58,5 & 22,40 & 35,30 \\
\hline $\begin{array}{l}\text { Akalan et al. } \\
2008\end{array}$ & 37 & $181,9^{*}$ & 63,00 & 34,0 & 1,720 & 81,9 * & 27,71 & 44,32 \\
\hline \multirow{4}{*}{$\begin{array}{l}\text { Ogawa et al. } \\
(1992)\end{array}$} & 14 & $195,0^{*}$ & $81,00^{\text {** }}$ & 23,0 & 1,645 & 57,7 & 21,30 & 37,00 \\
\hline & 14 & $164,0^{*}$ & 77,00 & 64,0 & 1,645 & 66,6 & 24,70 & 22,20 \\
\hline & 13 & $191,0^{*}$ & 66,00 & 26,0 & 1,666 & 55,4 & 19,90 & 52,10 \\
\hline & 13 & $169,0^{*}$ & 70,00 & 57,0 & 1,646 & 59,4 & 21,90 & 35,30 \\
\hline
\end{tabular}

Estimados empleando: * FC ${ }_{\text {Max mujeres }}=204,8-0,718$ (edad) $+0,162\left(\mathrm{FC}_{\text {Reposo }}\right)-0,105($ Peso corporal) - 6,2 x Código Fumador (1 fumador, 0 no fumador); ${ }^{*}{ }^{F} C_{\text {rep }}=15,3\left(\mathbf{H R}_{\max } / \mathrm{CAR}\right)$. 
Tabla 7b. Resultados de data antropomética y capacidad aeróbica, no fumadoras

\begin{tabular}{|c|c|c|c|c|c|c|c|c|c|}
\hline $\begin{array}{l}\text { MUJERES } \\
\text { FUENTE }\end{array}$ & $\mathrm{n}$ & $\begin{array}{c}\text { Frecuencia car- } \\
\text { diaca máxima } \\
\text { (latidos/min) }\end{array}$ & $\begin{array}{l}\text { Frecuencia } \\
\text { cardiaca } \\
\text { reposo } \\
\text { (latidos/min) }\end{array}$ & $\begin{array}{l}\text { Edad } \\
\text { (años) }\end{array}$ & $\begin{array}{l}\text { Talla } \\
(\mathrm{m})\end{array}$ & $\begin{array}{l}\text { Masa } \\
\text { corporal } \\
(\mathrm{kg})\end{array}$ & $\begin{array}{c}\text { Índice } \\
\text { de masa } \\
\text { corporal } \\
\left(\mathrm{kg} / \mathrm{m}^{2}\right)\end{array}$ & Fumador & $\begin{array}{c}\text { Capacidad } \\
\text { Aeróbica } \\
\text { Relativa } \\
\left(\mathrm{mlO}_{2} / \mathrm{kg}-\right. \\
\mathrm{min})\end{array}$ \\
\hline $\begin{array}{l}\text { Santos Silva } \\
\text { et al. (2018) }\end{array}$ & 695 & 195 & 81,1 & 16,0 & 1,613 & 58,5 & 22,4 & $\mathrm{NF}$ & 35,3 \\
\hline $\begin{array}{l}\text { Akalan et al. } \\
(2008)\end{array}$ & 37 & 182 & 63,0 & 34,0 & 1,720 & 82,0 & 27,7 & $\mathrm{NF}$ & 44,3 \\
\hline \multirow{4}{*}{$\begin{array}{l}\text { Ogawa et al. } \\
\text { (1992) }\end{array}$} & 14 & 195 & 81,0 & 23,0 & 1,645 & 57,7 & 21,3 & $\mathrm{NF}$ & 37,0 \\
\hline & 14 & 164 & 77,0 & 64,0 & 1,645 & 66,6 & 24,7 & $\mathrm{NF}$ & 22,2 \\
\hline & 13 & 191 & 66,0 & 26,0 & 1,666 & 55,4 & 19,9 & $\mathrm{NF}$ & 52,1 \\
\hline & 13 & 169 & 70,0 & 57,0 & 1,646 & 59,4 & 21,9 & $\mathrm{NF}$ & 35,3 \\
\hline & \multirow[t]{5}{*}{$\sum n=786$} & MEDIA & 73,0 & 36,7 & 1,7 & 63,3 & 23,0 & & 37,7 \\
\hline & & MEDIANA & 73,5 & 30,0 & 1,6 & 59,0 & 22,2 & & 36,2 \\
\hline & & MÁXIMO & 81,1 & 64,0 & 1,7 & 82,0 & 27,7 & & 52,1 \\
\hline & & MÍNIMO & 63,0 & 16,0 & 1,6 & 55,4 & 19,9 & & 22,2 \\
\hline & & \begin{tabular}{|l} 
DESV. \\
ESTANDAR
\end{tabular} & 7,8 & 19,5 & 0,0 & 9,9 & 2,8 & & 10,0 \\
\hline
\end{tabular}

\section{Función de regresión Tabla 7b - MUJERES NO FUMADORAS}

Capacidad aeróbica relativa $\left(\mathrm{mlO}_{2} / \mathrm{kg}-\mathrm{min}\right)=$

-181,4 - 0,4806 (Frecuencia cardíaca reposo, lat/min) - 0,1978 (Edad, años) + 161,7 (Talla, m)

- 1,626 (Masa corporal, kg) + 4,29 (Índice de masa corporal, kg/m²) (6)

\section{Comparación de las medianas del IMC para hombres, fumadores y no fumadores}

Hipótesis estadística $\boldsymbol{H}$ Kruskal-Wallis $\mathrm{H}_{0}: M d_{1}=M d_{2} ; \mathrm{H}_{1}: M d_{1} \neq M d_{2}$

\section{- Regla de decisión para este contraste:}

- $\quad$ Si $h_{\text {cálc }}<h_{\text {crit }}$ de Chi cuadrada con $\alpha ; v=k-1$. Aceptar $\mathrm{H}_{0}$

- $\quad$ Si $h_{\text {cálc }}>h_{\text {crit }}$ de Chi cuadrada con $\alpha ; v=k-1$. Rechazar $H_{0}$. (Walpole et al., 2012)

- $\quad$ Si $p$-valor $>\alpha=>$ Aceptar $\mathrm{H}_{0}$.

- $\quad$ Si $p$-valor $\leq \alpha=>$ Rechazar $\mathrm{H}_{0}$. (The Pennsylvania State University, 2017).

\section{- El valor Z}

Indica cómo el rango promedio para cada grupo se compara con el rango promedio de todas las observaciones. Se pueden interpretar los valores Z para cada grupo de la siguiente manera: 
- Cuanto más alto sea el valor absoluto, más lejos estará el rango promedio de un grupo del rango promedio general.

- Un valor $Z$ negativo indica que el rango promedio de un grupo es menor que el rango promedio general.

- Un valor Z positivo indica que el rango promedio de un grupo es mayor que el rango promedio general (Minitab Express Support, 2019).

Tabla 8. Prueba de Kruskal-Wallis, hombres no fumadores y fumadores vs mediana del índice de masa corporal (IMC), $\boldsymbol{\alpha}=0,05$ usando para la prueba de Kruskal-Wallis

\begin{tabular}{|l|c|c|c|c|}
\hline Sexo: HOMBRES & $\mathrm{n}$ & Mediana & $\mathrm{Z}$ & $\mathrm{p}$ \\
\hline No fumadores & 13 & 26,70 & 0,99 & 0,322 \\
\hline Fumadores & 7 & 25,77 & $-0,99$ & 0,322 \\
\hline
\end{tabular}

La Tabla 8 muestra el resultado de la prueba de Kruskal-Wallis con $\alpha=0,05$ para las medianas del Índice de Masa Corporal (IMC), siendo estas variables continuas vs hombres no fumadores/ fumadores siendo estas variables categóricas o cualitativas, de nivel dicotómico, en donde se indica el valor del nivel de significancia más bajo $p=0,322$ (Montgomery \& Runger, 2011).

La $\mathbf{p}=\mathbf{0 , 3 2 2} \geq \alpha=\mathbf{0 , 0 5}$ por lo que no se rechaza la hipótesis nula de que estadísticamente los IMC son iguales para $F y$ NF. Cuando $\mathrm{H}_{0}$ es verdadera, pero se rechaza, se comete el error Tipo I, al emplear una probabilidad dada de a (Freund \& Simon, 1992).

No fue posible comparar el IMC entre las mujeres $\mathrm{F}$ y las mujeres NF, debido que en el modelo del presente trabajo el número de observaciones a las mujeres NF fue muy pequeño.

Las contribuciones de esta investigación son: el delineado de la metodología para modelar las funciones de regresión y la construcción de tablas estratificadas categorizando data y resultados.

En el presente trabajo, para modelar las funciones multivariadas de regresión, se emplearon las siguientes variables de decisión o independientes: Frecuencia cardíaca reposo (latidos/min), Edad (años), Masa corporal $(\mathrm{kg})$, Índice de masa corporal $\left(\mathrm{kg} / \mathrm{m}^{2}\right)$ y la variable dependiente corresponde la Capacidad Aeróbica Relativa $\left(\mathrm{mlO}_{2}(\mathrm{~kg}-\mathrm{min})\right.$. 


\section{CONCLUSIONES}

- Fueron satisfechos el objetivo general y los objetivos específicos.

- La Hipótesis de investigación $\left(H_{l}\right)$ no puede ser rechazada.

- De acuerdo a la información recabada y presentada en las Tablas en este estudio, un fumador crónico, presumiblemente, presentaría una baja CAR.

- En este trabajo, se modelaron funciones multivariadas de regresión, que permiten determinar la CAR, para hombres y mujeres (fumadores y no fumadores), sin necesidad de ejercicio.

- La inspección ocular muestra en este estudio que el IMC de los NF es mayor que el de los F, pero con $\alpha=0,05$ la prueba de Kruskal-Wallis concluyó que no es estadísticamente significativa la diferencia entre los IMC de los NF vs los F.

- Las respuestas obtenidas al emplear las funciones de regresión vs mediciones directas fueron satisfactorias.

- Las variables consideradas fueron predictoras o independientes: Edad, Frecuencia Cardíaca en Reposo, Estatura, Masa Corporal e Índice de Masa Corporal; la variable de salida o dependiente es: la Capacidad Aeróbica Relativa.

En el presente trabajo las funciones de regresión modeladas mostraron que es adecuado su coeficiente de correlación calculado $\left(\mathrm{R}_{\text {calculado }}\right)$ :

$$
\begin{aligned}
n & =\text { observaciones en el modelo } \\
& =15+7+13+13+1+6=55 .
\end{aligned}
$$

Variables totales $(\mathrm{VT})=\quad(1$ variable dependiente $)+(5$ variables independientes $)=6$,

$$
\begin{aligned}
v & =\mathrm{GL}=\text { grados de libertad } \\
& =n-\mathrm{VT}=55-6=49 . \\
\alpha & =0,05 \text { nivel de significancia. }
\end{aligned}
$$

Según el software empleado la data que generó las funciones tiene $\mathbf{R}_{\text {calc }}{ }^{2}=86,69 \%=0,8669$, de donde $\mathbf{R}_{\text {calc }}=93,10 \%=0,9310>>$ que el valor crítico $R_{\text {tabulado }} \sim 0,46$ por lo tanto hay una excelente correlación.

Por todo lo anterior, son aceptables los correspondientes $R_{\text {calculado }}$ y $R_{\text {calculado }}^{2}$, estas funciones son un método preciso y sin ejercicios, alternativo a las medidas directas y útiles para: profesionales de la salud, reclutadores de: la fuerza laboral, fuerzas armadas, bomberos, deportistas, entre otros. 


\section{RECOMENDACIONES}

Realizar futuros estudios dentro de la misma línea de investigación del presente trabajo, pero con un mayor tamaño muestral para las mujeres fumadoras, que permitan modelar funciones de regresión con mejor fuerza de asociación.

\section{REFERENCIAS}

AKALAN, C., ROBERT, A., ROBERGS, R. A. y KRAVITZ, L. (2008). Prediction of $V_{2 \max }$ from an individualized submaximal cycle ergometer protocol. Journal of Exercise Physiology [en línea]. 11(2), 1-17. Disponible en: https://urlzs.com/SEfkc

ALADIN, A. I., AL RIFAI, M., RASOOL, S. H., KETEYIAN, S. J., BRAWNER, C. A. et al. (2015). The Association of Resting Heart Rate and Incident Hypertension: The Henry Ford Hospital Exercise Testing (FIT) Project Amer I. American Journal of Hypertension [en línea]. 29(2), 251257. Disponible en: https://doi.org/10.1093/ajh/hpv095.

AMERICAN HEART ASSOCIATION. All About Heart Rate (Pulse). [en línea]. 2019. Disponible en: https://urlzs.com/AvBX6

ANTOLINI, M. R. Physical fitness characteristics of an active firefighter population serving an urban area [en línea]. Trabajo fin de máster. Wilfrid Laurier University, 2014. Disponible en: https:// urlzs.com/uJuB3

ARIAS, F. G. El proyecto de investigación. Introducción a la metodología científica [en línea]. $6^{a}$ Edición. Ed. Episteme, C.A: Caracas, Venezuela, 2012. ISBN: 980-07-8529-9. Disponible en: https://urlzs.com/JfCmg

ARY, D., JACOBS, L. C. y RAZAVIEH, A. Introduction to Research in Education [en línea]. $2^{\text {a }}$. Edición. Ed. Holt, Rinehart and Winston: USA, 1979. ISBN O-03-020606-5. Disponible en: https:// urlzs.com/V73ME

ASH SCOTLAND. Cigarette smoking and body mass index (BMI). [en línea]. 2012. Disponible en: https://urlzs.com/s7MvJ

BANDYOPADHYAY, A. y BANDYOPADHYAY, P. (2007). Cardiorespiratory fitness in college students of Uttar Pradesh, India. Journal of Exercise Science and Physiotherapy [en línea]. 3: 38-41. Disponible en: https://urlzs.com/F49HM

BARRIOS, M., OCARANZA OZIMICA, J., LLACH FERNANDEZ, L., OSORIO FUENTEALBA, C., GINER COSTAGLIOLA, V. y SACOMORI, C. (2018). VO máximo indirecto y edad fitness de sedentarios y no sedentarios. Revista Internacional de Medicina y Ciencias de la Actividad Física y el Deporte [en línea].18(71), 493-505. Disponible en: http://dx.doi.org/10.15366/ rimcafd2018.71.006 
CARMONA, A. Aspectos Antropométricos de la Población Laboral Española, Aplicados al Diseño Industrial, 2003. ISBN 84-7425-655-0. Instituto Nacional de Seguridad e Higiene en el Trabajo. Madrid, España.

CHOI, B., STEISS, D., GARCÍA-RIVAS, J., KOJAKU, S., SCHNALL, P., DOBSON, M. Y BAKER, D. (2016). Comparison of body mass index with waist circumference and skinfoldbased percent body fat in firefighters: adiposity classification and associations with cardiovascular disease risk factors. International Archives of Occupational and Environmental Health [en línea]. 89, 435-448. Disponible en: https://doi.org/10.1007/s00420-015-1082-6

CROW, E. L., DAVIS, F. A. y MAXFIELD, M. W. Statistical Manual [en línea]. Dover Publications, Inc: New York. USA, 1960. ISBN: 486-60599-X. Disponible en: https://urlzs.com/EF1bf

DEHNEH, N., RAJAB, M., ZARZOUR, W. y JOUMA, M. (2016). Profile of healthy female students in Syria: Investigation on anthropometric, lifestyle, and dietary characteristics, along with hematological and plasma biochemical parameters. Cogent Medicine [en línea]. 3(1), 1180741. Disponible en: https://doi.org/10.1080/2331205X.2016.1180741

EKBLOM-BAK, E., HELLENIUS, M. L., EKBLOM, Ö., ENGSTRÖM, L.-M. y EKBLOM, B. (2009). Fitness and abdominal obesity are independently associated with cardiovascular Risk. Journal of Internal Medicine [en línea]. 266(6), 547-557. Disponible en: https://doi.org/10.1111/j.13652796.2009.02131.x

EROĞLU, H. y YÜKSEK, S. (2018). The Effect of Smoking on the Physical Fitness of Elderly Male Subjects. Universal Journal of Educational Research [en línea]. 6(6): 1158-1166. Disponible en: https://doi.org/10.13189/ujer.2018.060605

FERNÁNDEZ, J.A., RAMOS, H.S., SANTAMARÍA, O. y RAMOS S. (2018). Relación entre consumo de oxígeno, porcentaje de grasa e índice de masa corporal en universitarios. Hacia la Promoción de la Salud [en línea]. 23(2), 79-89. Disponible en: http://dx.doi.org/10.17151/ hpsal.2018.23.2.6.

FOLLMER GREENHOOT, A. y DOWSETT, C. J. (2012). Secondary Data Analysis: An Important Tool for Addressing Developmental Questions. Journal of Cognition and Development [en línea]. 13(1), 2-18. Disponible en: https://doi.org/10.1080/15248372.2012.646613

FREUND, J. E. y SIMON, G. A. Modern Elementary Statistics. $8^{\circ}$ Ed. Prentice Hall. New Jersey. USA, 1992. ISBN 0-13-602699-0

GEORGE, J. D.; GARTH F., A. y VEHRS, P. R. Tests y pruebas físicas. 4a Edición. Editorial Paidotribo, España, 2007

GONZÁLEZ, A. y ACHIARDI, O. (2016). Relación entre capacidad aeróbica y variables antropométricas en mujeres jóvenes físicamente inactivas de la ciudad de Concepción, Chile. Revista Chilena de Nutrición [en línea]. 43(1), 18-23. Disponible en: http://dx.doi.org/10.4067/ S0717-75182016000100003

GRIGALIŪNIENĖ, A., RAMONAS, A., ČELUTKIENĖ, J., ŠILEIKIENĖ, V., RUDYS, A., JUOCEVIČIUS, A. y LAUCEVIČIUS, A. (2013). Cardiorespiratory Parameters of Exercise Capacity in a Healthy Lithuanian Population: The Pilot Study. Hellenic Journal Cardiology [en 
línea]. 54,107-118. Disponible en: https://urlzs.com/KKbTH

HEYWARD, V. H. Advanced Fitness Assessment and Exercise Prescription. $6^{\circ}$ Edition. Human Kinetics. Champaign II. USA, 2010. ISBN-10: 0736086595.

INBAR, O. OTEN, A., SCHEINOWITZ, M., ROTSTEIN, A., DLIN, R. y CASABURI, R. (1994). Normal cardiopulmonary responses during incremental exercise in 20-70-yr-old men. Med Sci Sport Exerc. [en línea]. 26(5), 538-546. Disponible en: https://doi.org/10.1249/00005768199405000-00003

KENNEDY, J. B. y NEVILLE, A. M. Basic statistical methods for engineers \& scientists. 2nd Edition. Edit. A Dun-Donnelley. New York, USA, 1976. ISBN: 0-7002-2480-7.

LEÓN PÉREZ, J. M., RUEDA MÉNDEZ, S. y MUNDUATE JACA, M. L. Psicología de los Recursos Humanos [en línea]. Universidad de Sevilla, España, 2007. ISBN 978-84-691-1948-8. Disponible en: https://urlzs.com/GPM9B

LEY PARA LAS PERSONAS CON DISCAPACIDAD, 2007. Gaceta Oficial 38.598 [en línea]. Disponible en: https://urlzs.com/ie9Tx

LOPCYMAT (2005). Ley Orgánica de Prevención, Condiciones y Medio Ambiente de Trabajo [en línea]. Gaceta Oficial № 38.236 del 26 de julio de 2005. Disponible en: https://urlzs.com/R7yq5

MINISTERIO DE SALUD SUBSECRETARÍA DE SALUD PÚBLICA, CHILE. Guía Clínica Examen de medicina preventiva, 2009 [en línea]. ISBN: 978-956-8823-01-6. Disponible en: https://urlzs. com/Q7MjS

MINITAB EXPRESS SUPPORT. Interpret all statistics and graphs for Kruskal-Wallis Test, 2019 [en línea]. Disponible en: https://urlzs.com/4gve6

MIYATAKE, N., NUMATA, T., CAO, Z. B., MIYACHI, M. y TABATA, I. (2012). Relationship between predicted oxygen uptake and cigarette smoking in Japanese men. Age [en línea]. 44(14.1), 21. Disponible en http://dx.doi.org/10.4236/health.2012.47066.

MONTGOMERY, D.C, y RUNGER, G.C. Probabilidad y estadística aplicada a la ingeniería. 2a Edición. México. Limusa Wiley, 2011. ISBN: 978-968-18-5915-2.

NEALEN, P. M. Exercise and lifestyle predictors of resting heart rate in healthy young adults, 2016 [en línea]. Disponible en: https://doi.org/10.14198/jhse.2016.113.02.

OGAWA, T., SPINA, R. J., MARTIN 3RD, W. H., KOHRT, W. M., SCHECHTMAN, K. B., HOLLOSZY, J. O. y EHSANI, A. A. (1992). Effects of aging, sex, and physical training on cardiovascular responses to exercise. Circulation [en línea]. 86(2), 494-503.Disponible en: https://urlzs.com/Gx9RY

PALELLA, S. y MARTINS, F. Metodología de la Investigación Cuantitativa. FEDUPEI. Venezuela, 2010. ISBN 980-273-445-4.

PAPATHANASIOU, G., GEORGAKOPOULOS, D., PAPAGEORGIOU, E., ZERVA, E., MICHALIS, L., KALFAKAKOU, V. y EVANGELOU, A. (2013). Effects of smoking on heart rate at rest and during exercise, and on heart rate recovery, in young adults. Hellenic J Cardiol [en línea]. 54(3), 
168-177. Disponible en: https://urlzs.com/9bn8i

PARACHA, P.I., KHAN, T., VRIESEKOOP, F., SALAHUDDIN, M., PARACHA, S.I., UDDIN, Z., ULLAH, N. y ASIF, Y. (2015). Association between smoking and anthropometric characteristics, biochemical markers, and dietary intake of Pakistani male adult population. Advances in Food Technology and Nutritional Sciences-Open Journal [en línea]. 1(4), 88-96. Disponible en: https:/l urlzs.com/Tydcd

POURTAGHI, G., VALIPOUR, F., SADEGHIALAVI, H. \& LAHMI, MA. Anthropometric characteristics of Iranian military personnel and their Changes over Recent Years [en línea]. 5(3); July, 2014. ISSN 20086814, 20086520. [Consultado 28 diciembre 2018]. Disponible en: https://urlzs.com/mWxbU

RAHIMI, N. A., SEDEK, R., TEH, A. H. y HARUN, M. S. (2017). Relationship Between Body Composition and Physical Fitness of Rescue Firefighter Personnel in Selangor, Malaysia. Pakistan Journal of Nutrition [en línea]. 16,77-83. Disponible en: https://doi.org/10.3923/pjn.2017.77.83.

RAMIREZ-VELEZ, R., ARGOTHYD, R., MENESES-ECHAVEZ, J. F., SANCHEZ-PUCCINI, M. B., LOPEZ-ALBAN, C. A. Y COHEN, D. D. (2014). Anthropometric Characteristics and Physical Performance of Colombian Elite Male Wrestlers. Asian journal of sports medicine [en línea]. 5(4). Disponible en: https://doi.org/10.5812/asjsm.23810.

RAHIMI, N. A., SEDEK, R., TEH, A. H. y HARUN, M. S. (2017). Relationship Between Body Composition and Physical Fitness of Rescue Firefighter Personnel in Selangor, Malaysia. Pakistan Journal of Nutrition [en línea]. 16,77-83. Disponible en: https://doi.org/10.3923/pjn.2017.77.83.

ROBERGS, R. A. y LANDWEHR, R. (2002). The surprising history of the "HR ${ }_{\max }=220$-age" equation. Journal of Exercise Physiology Online [en línea]. 5(2), 1-10. Disponible en: https://urlzs. com/NRRjs

RUIZ, R. J., PIRES, P. F., PELAI, E. B., DE CASTRO, E. M., MESCOLLOTTO, F. F., MORENO, M. A., y BIGATON, D. R. (2019). Acute effect of aerobic and resistance exercises on cardiovascular and neuromuscular responses in normotensive. Manual Therapy, Posturology \& Rehabilitation Journal [en línea]. 16, 0-0. Disponible en: https://urlzs.com/eSLHQ

SANTOS SILVA, D. A., RODRIGUES DELIMA, T., y AND TREMBLAY, M. S. (2018). Association between Resting Heart Rate and Health-Related Physical Fitness in Brazilian Adolescents. BioMed Research International [en línea]. 2018 (article ID 3812197). Disponible en: https://doi. org/10.1155/2018/3812197

SECCHI, J.D. y GARCÍA, G.C. (2013). Aptitud física cardiorrespiratoria y riesgo cardiometabólico en personas adultas jóvenes. Revista Española de Salud Pública [en línea]. 87(1), 35-48. Disponible en: https://urlzs.com/AEBwD

SENGUPTA, P. y KRAJEWSKA-KULAK, E. (2014). Evaluation of physical fitness and weight status among fisherwomen in relation to their occupational workload. Journal of Epidemiology and Global Health [en línea]. 4, 261-268. Disponible en: https://doi.org/10.1016/j.jegh.2014.03.005.

SHARKEY, B. J. y DAVIS, P. O. Hard Work. Defining Physical Work Performance Requirements. Human Kinetics, 2008. EE.UU. ISBN 10: 0-7360-6536-9 
SHARMA, M., KAMAL, R.B. y CHAWLA, K. (2016). Correlation of body composition to aerobic capacity: A cross sectional study. International Journal of Applied Research [en línea]. 2(1), 3842. Disponible en: https://urlzs.com/YgmbH

SHEPHARD, R.J. y PIMM, P. (1975). Physical fitness of Canadian physical education students with a note on international differences [en línea]. British Journal of Sports Medicine [en línea]. 9(4), 165-174. Disponible en: https://urlzs.com/vn8HH

STEPHENS, P. Fitness evaluation of Gaelic football players. 2004. Tesis Doctoral. Dublin City University. Disponible en: https://urlzs.com/GGaAT

STRASSMANN, A., STEURER-STEY, C. D, LANA, C.D., ZOLLER, M., TURK, A. J., SUTER, P y PUHAN, M. A. (2013). Population-based reference values for the 1-min sit-to-stand test. International journal of public health [en línea]. 58(6), 949-953. Disponible en: https://doi. org/10.1007/s00038-013-0504-z .

THE PENNSYLVANIA STATE UNIVERSITY. 3.2 - Hypothesis Testing (P-value approach) [en línea]. 2017. Disponible en: https://urlzs.com/dMGf4

UTH, N.; SORENEN, H.; OVERGAARD, K. y PEDERSEN, P.K. (2004). Estimation of VंO $2 m a x$ from the ratio between HR max and HR rest-the Heart Rate Ratio Method. Eur J Appl Physiol [en línea]. 91(1), 111-115. Disponible en: https://doi.org/10.1007/s00421-003-0988-y

VILLARROEL, V., MOLINA, C. M., DÁVILA, D. y ARATA-BELLABARBA, G. (2004). Efectos del hábito de fumar sobre la concentración de norepinefrina, la presión arterial y el pulso en hombres jóvenes. Revista Venezolana de Endocrinología y Metabolismo [en línea]. 2(1), 24-28. Disponible en: https://urlzs.com/reJ34

WALPOLE, R. E., MYERS, R. H., MYERS, S. L. yYE, K. Probabilidad y Estadística para Ingeniería y Ciencias. 9a Edición, 2012. ISBN 978-607-32-1417-9. Pearson. México.

WHALEY, M. H., KAMINSKY, L. A., DWYER G. B., GETCHELL, L. H. y NORTON, J.A. (1992). Predictors of over- and underachievement of age-predicted maximal heart rate. Med Sci Sports Exerc. [en línea]. 24(10),1173-9. Disponible en: https://urlzs.com/cZd7A

WIEDENHÖFER S, H. Pruebas no paramétricas para las ciencias agropecuarias. Muestras pequeñas. Instituto Nacional de Investigaciones Agrícolas. Publicación técnica. Maracay, Venezuela, 1993. ISBN 980-318-040-X. 


\section{Anexo 1}

Valores del coeficiente de correlación $r$ Funciones bivariantes y multivariantes

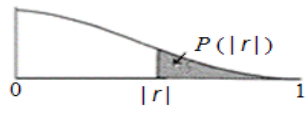

\begin{tabular}{|c|c|c|c|c|c|c|c|c|c|c|}
\hline \multicolumn{5}{|c|}{5 Porciento de nivel de significancia } & \multirow[b]{3}{*}{6} & \multicolumn{5}{|c|}{1 Porciento de nivel de significancia } \\
\hline \multicolumn{5}{|c|}{$\mathrm{N}^{\circ}$ total de variables $=$ Variable dependiente + variables independientes } & & \multicolumn{5}{|c|}{$\mathrm{N}^{\circ}$ total de variables $=$ Variable dependiente + variables independientes } \\
\hline$v$ & 2 & 3 & 4 & 5 & & 2 & 3 & 4 & 5 & $v$ \\
\hline 1 & 0,997 & 0,999 & 0,999 & 0,999 & & 1,000 & 1,000 & 1,000 & 1,000 & 1 \\
\hline 2 & 0,950 & 0,975 & 0,983 & 0,987 & & 0,990 & 0,995 & 0,997 & 0,998 & 2 \\
\hline 3 & 0,878 & 0,930 & 0,950 & 0,961 & & 0,959 & 0,976 & 0,983 & 0,987 & 3 \\
\hline 4 & 0,811 & 0,881 & 0,912 & 0,930 & & 0,917 & 0,949 & 0,962 & 0,970 & 4 \\
\hline 5 & 0,754 & 0,836 & 0,874 & 0,898 & & 0,874 & 0,917 & 0,937 & 0,949 & 5 \\
\hline 6 & 0,707 & 0,795 & 0,839 & 0,867 & & 0,834 & 0,886 & 0,911 & 0,927 & 6 \\
\hline 7 & 0,666 & 0,758 & 0,807 & 0,838 & & 0,798 & 0,855 & 0,885 & 0,904 & 7 \\
\hline 8 & 0,632 & 0,726 & 0,777 & 0,811 & & 0,765 & 0,827 & 0,860 & 0,882 & 8 \\
\hline 9 & 0,602 & 0,697 & 0,750 & 0,786 & & 0,735 & 0,800 & 0,836 & 0,861 & 9 \\
\hline 10 & 0,576 & 0,671 & 0,726 & 0,763 & & 0,708 & 0,776 & 0,814 & 0,840 & 10 \\
\hline 11 & 0,553 & 0,648 & 0,703 & 0,741 & & 0,684 & 0,753 & 0,793 & 0,821 & 11 \\
\hline 12 & 0,532 & 0,627 & 0,683 & 0,722 & & 0,661 & 0,732 & 0,773 & 0,802 & 12 \\
\hline 13 & 0,514 & 0,608 & 0,664 & 0,703 & & 0,641 & 0,712 & 0,755 & 0,785 & 13 \\
\hline 14 & 0,497 & 0,590 & 0,646 & 0,686 & & 0,623 & 0,694 & 0,737 & 0,768 & 14 \\
\hline 15 & 0,482 & 0,574 & 0,630 & 0,670 & & 0,606 & 0,677 & 0,721 & 0,752 & 15 \\
\hline 16 & 0,468 & 0,559 & 0,615 & 0,655 & & 0,590 & 0,662 & 0,706 & 0,738 & 16 \\
\hline 17 & 0,456 & 0,545 & 0,601 & 0,641 & & 0,575 & 0,647 & 0,691 & 0,724 & 17 \\
\hline 18 & 0,444 & 0,532 & 0,587 & 0,628 & & 0,561 & 0,633 & 0,678 & 0,710 & 18 \\
\hline 19 & 0,433 & 0,520 & 0,575 & 0,615 & & 0,549 & 0,620 & 0,665 & 0,698 & 19 \\
\hline 20 & 0,423 & 0,509 & 0,563 & 0,604 & & 0,537 & 0,608 & 0,652 & 0,685 & 20 \\
\hline 21 & 0,413 & 0,498 & 0,552 & 0,592 & & 0,526 & 0,596 & 0,641 & 0,674 & 21 \\
\hline 22 & 0,404 & 0,488 & 0,542 & 0,582 & & 0,515 & 0,585 & 0,630 & 0,663 & 22 \\
\hline 23 & 0,396 & 0,479 & 0,532 & 0,572 & & 0,505 & 0,574 & 0,619 & 0,652 & 23 \\
\hline 24 & 0,388 & 0,470 & 0,523 & 0,562 & & 0,496 & 0,565 & 0,609 & 0,642 & 24 \\
\hline 25 & 0,381 & 0,462 & 0,514 & 0,553 & & 0,487 & 0,555 & 0,600 & 0.633 & 25 \\
\hline 26 & 0,374 & 0,454 & 0,506 & 0,545 & & 0,478 & 0,546 & 0,590 & 0,624 & 26 \\
\hline 27 & 0,367 & 0,446 & 0,498 & 0,536 & & 0,470 & 0,538 & 0,582 & 0,615 & 27 \\
\hline 28 & 0,361 & 0,439 & 0,490 & 0,539 & & 0,463 & 0,530 & 0,573 & 0,606 & 28 \\
\hline 29 & 0,355 & 0,432 & 0,482 & 0,521 & & 0,456 & 0,522 & 0,565 & 0,598 & 29 \\
\hline 30 & 0,349 & 0,426 & 0,476 & 0,514 & & 0,449 & 0,514 & 0,558 & 0,591 & 30 \\
\hline 35 & 0,325 & 0,397 & 0,445 & 0,482 & & 0,418 & 0,481 & 0,523 & 0,556 & 35 \\
\hline 40 & 0,304 & 0,373 & 0,419 & 0,455 & & 0,393 & 0,454 & 0,494 & 0,526 & 40 \\
\hline 45 & 0,288 & 0,353 & 0,397 & 0,432 & & 0,372 & 0,430 & 0,470 & 0,501 & 45 \\
\hline 50 & 0,273 & 0,336 & 0,379 & 0,412 & 0,46 & 0,354 & 0,410 & 0,449 & 0,479 & 50 \\
\hline 60 & 0,250 & 0,308 & 0,348 & 0,380 & & 0,325 & 0,377 & 0,414 & 0,442 & 60 \\
\hline 70 & 0,232 & 0,286 & 0,324 & 0,354 & & 0,302 & 0,351 & 0,386 & 0,413 & 70 \\
\hline 80 & 0,217 & 0,269 & 0,304 & 0,332 & & 0,283 & 0,330 & 0,362 & 0,389 & 80 \\
\hline 90 & 0,205 & 0,254 & 0,288 & 0,315 & & 0,267 & 0,312 & 0,343 & 0,368 & 90 \\
\hline 100 & 0,195 & 0,241 & 0,274 & 0,300 & & 0,254 & 0,297 & 0,327 & 0,351 & 100 \\
\hline 125 & 0,174 & 0,216 & 0,246 & 0,269 & & 0,228 & 0,266 & 0,294 & 0,316 & 125 \\
\hline 150 & 0,159 & 0,198 & 0,225 & 0,247 & & 0,208 & 0,244 & 0,270 & 0,290 & 150 \\
\hline 200 & 0,138 & 0,172 & 0,196 & 0,215 & & 0,181 & 0,212 & 0,234 & 0,253 & 200 \\
\hline 300 & 0,113 & 0,141 & 0,160 & 0,176 & & 0,148 & 0,174 & 0,192 & 0,208 & 300 \\
\hline 400 & 0,098 & 0,122 & 0,139 & 0,153 & & 0,128 & 0,151 & 0,167 & 0,180 & 400 \\
\hline 500 & 0,088 & 0,109 & 0,124 & 0,137 & & 0,115 & 0,135 & 0,150 & 0,162 & 500 \\
\hline 1000 & 0,062 & 0,077 & 0,088 & 0,097 & & 0,081 & 0,096 & 0,106 & 0,116 & 1000 \\
\hline
\end{tabular}

The critical value of $r$ at a given level of significance, total number of variables, and degrees of freedom $v$, is read from the table. If the compute $|r|$ exceeds the critical value, then the null hypothesis that there is no association between the variables is rejected at the given level. The test is an equal-tails test, since we are usually interested in either positive or negative correlation. The shaded portion of the figure is the stipulated probability as a level of significance.

Fuente: Table reproduced by Kennedy \& Neville (1976), with the permission of the authors and publisher from Crow et al. (1960). Statistical Manual. New York: Dover Publications, Inc. (http://www.dtic.mil/dtic/tr/fulltext/u2/149334.pdf ) 